University of Louisville

ThinkIR: The University of Louisville's Institutional Repository

\title{
$5-2015$
}

\section{Role of ribosomal biogenesis in axonal morphogenesis.}

Nicholas McChesney Lynch

University of Louisville

Follow this and additional works at: https://ir.library.louisville.edu/honors

Part of the Biochemistry, Biophysics, and Structural Biology Commons, and the Cell and Developmental Biology Commons

\section{Recommended Citation}

Lynch, Nicholas McChesney, "Role of ribosomal biogenesis in axonal morphogenesis." (2015). College of Arts \& Sciences Senior Honors Theses. Paper 58.

http://doi.org/10.18297/honors/58

This Senior Honors Thesis is brought to you for free and open access by the College of Arts \& Sciences at ThinkIR: The University of Louisville's Institutional Repository. It has been accepted for inclusion in College of Arts \& Sciences Senior Honors Theses by an authorized administrator of ThinkIR: The University of Louisville's Institutional Repository. This title appears here courtesy of the author, who has retained all other copyrights. For more information, please contact thinkir@louisville.edu. 
Role of Ribosomal Biogenesis in Axonal Morphogenesis

By

Nicholas McChesney Lynch

Submitted in partial fulfillment of the requirements

for Graduation summa cum laude

University of Louisville

March 30, 2015 


\section{Role of ribosomal biogenesis in axonal morphogenesis}

Nicholas Lynch

University of Louisville

Thesis Advisor: Dr. Michal Hetman

Keywords: nucleolus, neurites, ribosomal proteins, axons, RPS6, TIF-IA, shRNA, B23/NPM1

\section{ABSTRACT}

Elongating axons require a host of macromolecules for outgrowth. The importance of lipid, protein, and general RNA synthesis for initial axonal growth has been previously investigated, but the role of rRNA synthesis and ribosome production in axonal morphogenesis remains unclear. Therefore, rat hippocampal neurons were cultured and transfected with shRNA against either TIF-IA (the activator of RNA polymerase I) or RPS6 (a small ribosomal subunit component) to determine the effects of impaired de novo ribosome synthesis on axonal development during the first three days after plating. Our data show that shRPS6 weakly inhibited total axon length, but not longest axon path or number of axonal branches; shTIF-IA showed no significant inhibitory effect in all three parameters. Thus, these results indicate that the pre-existing ribosome supply was at least temporarily sufficient for growth during this initial phase of axonal development.

\section{BACKGROUND}

Known as the cellular "ribosome factory", the nucleolus is the dynamic sub-nuclear structure in all eukaryotic cells. The nucleolus is composed of RNA and proteins grouped around specific chromosomal regions called nucleolar organizing regions (NORs), which are comprised of numerous tandem repeats of the ribosomal DNA (rDNA) coding for ribosomal RNA (rRNA) [1]. Primarily involved in ribosomal biogenesis, the nucleolus directs the synthesis and processing of rRNAs before assembling them together with ribosomal proteins (RPs) imported from the cytoplasm to create the large $60 \mathrm{~S}$ and small $40 \mathrm{~S}$ ribosomal subunits found in eukaryotic ribosomes [2]. Following production in the nucleolus and maturation in the nucleus, the large $60 \mathrm{~S}$ and small 40S ribosomal subunits are transported out of the nucleus and into the cytoplasm, where they then combine with processed mRNA transcripts. Specifically, the 40S subunit first binds with translation initiation factors and an mRNA transcript, which then facilitates the binding of the 60S subunit [3]. This constitutes the formation of a fully functional ribosome, which is the cellular translation machinery that is responsible for catalyzing protein synthesis [4]. Given the multitude of proteins required for normal cell function and survival, the role of ribosomes as the cellular translation machinery makes them indispensable for cellular growth. As such, the critically important roles that the nucleolus plays in ribosomal biogenesis can have a drastic impact on the capacity of the cell to grow [2]. Additionally, the nucleolus has been attributed many roles in the maintenance of cellular homeostasis and its dysfunction has been linked to various pathologies [5]. While in eukaryotes RNA polymerase (pol) II is responsible for the transcription of genes encoding proteins, RNA pol I is responsible for the transcription of rRNA-encoding genes in the nucleolus [6,37]; therefore, RNA pol I transcriptional activity 
serves as an important regulator of ribosomal biogenesis and thus enables protein synthesis and cellular growth [7]. Activity of RNA pol I, in turn, is closely regulated by multiple biochemical pathways to adjust ribosomal biogenesis according to cellular demands [7]. Generally speaking, conditions that are detrimental to cell growth (such as lack of growth factors or inhibition of protein synthesis) result in a down-regulation of RNA pol I [30]. Conversely, RNA pol I is upregulated to increase rRNA synthesis by the presence of neurotrophic factors, mitogenic signals, and favorable environmental conditions. Specifically, as one of its final targets, the extracellular signal-related kinase (ERK) pathway phosphorylates transcription initiation factor 1A (TIF-IA), which promotes the association of RNA pol I with rDNA gene promoters in rRNA synthesis [31]. In sum, RNA pol I tightly links cellular growth to changes in environmental conditions by determining the level of ribosome production [30-32].

Given its role in the regulation of RNA pol I activity, TIF-IA was selected as a target for shRNA-mediated inhibition in this experiment. More than two decades ago, TIF-IA was found to control the growth-dependent regulation of rRNA synthesis in mice by responding to extracellular signals [38]. More broadly, it was found that TIF-IA is the mammalian functional homolog of the yeast factor Rrn3p, both of which "activate" RNA pol I, making it competent for transcription initiation [39, 40]. Specifically, this activation is achieved by direct association of TIF-IA with RNA pol I to form a transcriptionally active holoenzyme that is able to bind to the rDNA promoter and initiate transcription in the presence of auxiliary cofactors, including the selectivity factor SL-1 (also called TIF-IB) and upstream binding factor (UBF) [38]. Different signaling pathways are believed to be responsible for the ability of TIF-IA to respond to extracellular signals and regulate cell growth and proliferation, especially via ERK- and mTOR (mammalian target of rapamycin)-mediated modulation of the phosphorylation state of TIF-IA $[31,32]$. Further regulation includes the action of the Akt pathway, which stabilizes TIF-IA, triggers its nucleolar translocation, and augments its interaction with RNA pol I [41]. Also, in connection with TIF-IA, this project takes advantage of the nucleolar phosphoprotein B23 (also known as numatrin, nucleophosmin, and NPM1) [18]. Given its localization in the nucleolus and its association with pre-ribosomal particles, B23 is an established nucleolar marker [18, 24]. As such, B23 dispersion serves as an indicator of nucleolar disruption and, thus, successful shTIFIA inhibition of RNA pol I activity. In sum, as the primary regulator of RNA pol I-driven rDNA transcription, TIF-IA activity is of obvious importance for ribosomal biogenesis.

Despite shared transcriptional and translational processes underlying general cellular growth, different cells types demonstrate different patterns of development based on their specifically determined functions. Neurons are post-mitotic cells that no longer divide but continue to grow. Specifically, this growth consists of the development and elongation of neurites, including axons and dendrites. This neurite development - specifically with hippocampal neuron cultures - has been thoroughly studied in vitro, elucidating a process that can be broken down into five characteristic stages: within hours of adhering to the substrate, Stage 1 cells develop many small, motile, peripheral extensions known as lamellipodia; during Stage 2 the lamellipodia extend and become minor processes after about one day in vitro (DIV 1); within DIV 1-2, Stage 3 sees the particularly pronounced outgrowth of a single minor process, which forms a single major process. This major process continues its rapid growth, becoming a clearly identifiable axon between DIV 3-7. Significant dendritic outgrowth begins during Stage 4 around DIV 4, but the dendrites remain morphologically and biochemically distinct from the singular axon. [23] 
However, such extensive morphological development of neurons involves a marked increase in cell volume and surface area. These increases necessarily require various macromolecules to support membranous and cytoskeletal expansions. An important study by Jareb and Banker demonstrated how axonal growth is differentially affected by inhibition of the supply of these macromolecules in rat hippocampal neurons at 18 hours after plating: disruption of the supply of Golgi-derived vesicles by treatment with brefeldin A inhibited axonal growth within one hour, showing the importance of lipid membrane components; cycloheximide (CHX)-mediated disruption of protein synthesis also significantly inhibited axonal growth, but only after six hours; and actinomycin D, a general RNA synthesis inhibitor, also inhibited axonal growth after nine hours [42] (Figure 1). The selected timeframe of about 18-30 hours after plating roughly corresponds to the aforementioned Stage 3 (DIV 1-2) in which robust axon growth begins [23]. Therefore, the supply of lipids, RNA, and proteins to the developing axon appear to be crucial for growth during this early phase of elongation. Of particular interest for this work is the necessity of protein synthesis for axonal growth, given that ribosomal biogenesis ultimately serves to produce ribosomes capable of fulfilling the cell's translational needs.

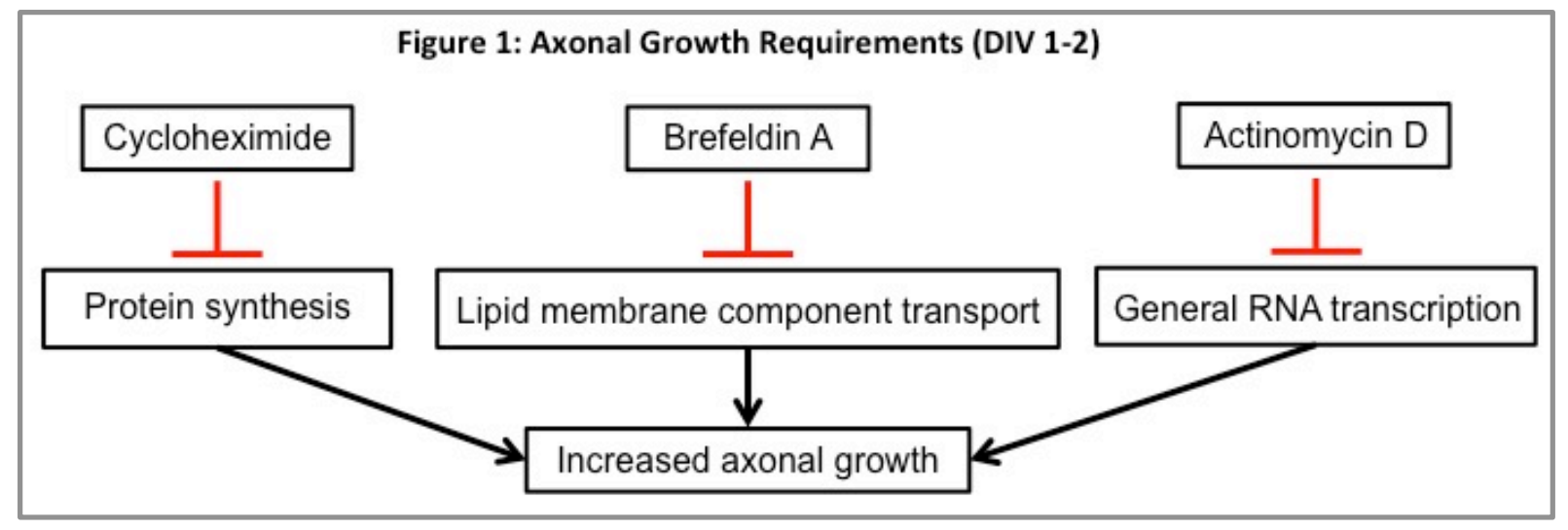

Given the importance of protein synthesis for axonal development, proper regulation becomes crucial. The protein kinase mTOR has been identified as a key regulator of cellular growth via its regulation of protein synthesis and the translation machinery [29]. In particular, PI3K (phosphatidylinositol 3-kinase)/mTOR signaling regulates TIF-IA activity [7,32]. In addition to affecting numerous other downstream targets, the role of $\mathrm{mTOR}$ in recruitment of ribosomes to mRNA has been linked to its phosphorylation of RPS6, a functional component of ribosomes [12]. The aforementioned interlacing signaling pathways provide for careful control of protein synthesis. Nevertheless, the location of this protein synthesis within the neuron has been debated for decades. While dendrites have long been known to contain ribosomes and undergo local protein synthesis, the same was not thought to occur in axons [28]. In fact, ribosomes were thought to be excluded from axons; it was believed that axoplasmic proteins were actually produced in the cell body and slowly transported to their particular destination(s) in the axon [33, and references contained therein]. However, a recently growing body of evidence has indicated that axons also possess ribosomes and rely on local protein synthesis for growth and directional responses [34 and references contained therein]. Indeed, studies have shown that ribosomes exist within periaxoplasmic ribosomal plaques (PARPs) distributed across the length of the axon, and that myosin and kinesin motor proteins play a role in this ribosomal trafficking and distribution [43]. Local protein synthesis in axons is thought to be necessary for expedient control of the 
local proteome, including correct localization of axonal proteins, allowing the growth cone to quickly respond to environmental stimuli, and to avoid macromolecular crowding caused by protein storage [35 and references contained therein]. Interestingly, it has been shown that neurite outgrowth is resistant to incomplete inhibition of protein synthesis by CHX; this resistance is attributed to a concurrent decrease in the rate of protein degradation that parallels the decrease in protein synthesis [36].

Observations of nucleolar morphology in different regions of the nervous system have provided evidence that increased ribosomal biogenesis - as indicated by dynamic nucleolar activity and reorganization - is directly linked to neuronal growth [8]. Indeed, Dr. Hetman's lab has demonstrated that experimental knockdown of TIF-IA - a coactivator of RNA pol I - in rat hippocampal neuron cultures inhibits brain-derived neurotrophic factor-induced (BDNF) neurite growth, although not overall protein synthesis [7]. These results demonstrate the dependence of nervous system development on the nucleolar transcriptional activity of RNA pol I, indicating the importance of the nucleolus for neural cell growth. Because general protein synthesis was not reduced in experiments with cells from DIV 2-3, these morphogenetic requirements for neuritic growth in this early phase of development cannot be accounted for simply in terms of changes in overall capacity for translation. By contrast, when RNA pol I was inhibited on DIV 6-8, translational capacity was clearly reduced [Slomnicki and Hetman, unpublished observations]. Stemming from this research on the role of RNA pol I in neurite outgrowth, the Hetman lab is now investigating the role of ribosomal biogenesis in neurite outgrowth via short hairpin RNA (shRNA) knockdown of TIF-IA and various ribosomal proteins, including RPS6, RPL4, and RPS 14. Currently unpublished data from Dr. Hetman's lab has shown that shRNA-mediated knockdown of TIF-IA, RPL4, RPS14, and RPS6 significantly reduced dendritic growth in later stages from DIV 6-9; this indicates the importance of pol I activity and ribosomal biogenesis in dendritic growth [Slomnicki et al. In preparation]. However, these experiments did not involve axons, which, unlike dendrites, are uniquely neuronal structures; therefore, it remains to be tested whether or not shRNA knockdown of TIF-IA and/or ribosomal proteins will similarly impact axonal growth.

As integral components of functioning ribosomes, ribosomal proteins are important for protein synthesis and, thus, cellular growth. For this experiment, RPS6 was selected as the ribosomal target for shRNA knockdown to determine the effect that this would have on axonal growth. RPS6 was discovered in 1974 in connection with liver regeneration [10]. RPS6 is a ribosomal protein consisting of 249 amino acids, including five known phosphorylation sites - which are evolutionarily conserved in metazoans - at clustered residues in the C-terminal region of the protein: $\operatorname{Ser}^{235}, \operatorname{Ser}^{236}, \operatorname{Ser}^{240}, \operatorname{Ser}^{244}$, and $\operatorname{Ser}^{247}$ [11]. Since its discovery, RPS6 has been one of the most extensively researched ribosomal proteins, largely due to the fact that it was the first ribosomal protein to be known for its capacity for inducible phosphorylation [12]. In terms of its canonical role as a ribosomal protein, RPS6 is a component of the small 40S subunit of eukaryotic ribosomes; it is located at the interface of the 40S subunit with the 60S subunit, where it interacts with initiation factors, mRNA, and tRNA [13]. As such, it appears that RPS6 functions in mRNA binding, and so phosphorylation of RPS6 may help to regulate translation [12]. With respect to phosphorylation, RPS6 is included within the category of S6 kinase (S6K) substrates [12]. As such, it was previously suggested that RPS6 phosphorylation may regulate initiation of translation and inclusion of ribosomes in polysomes; however, experiments with 
RPS6 $^{P-/-}$ knock-in mice liver cells (with phosphorylation prevented at all aforementioned serine residues) have shown the same proportion of ribosome recruitment into polysomes as in the liver cells of wild-type mice. Indeed, in comparison with the wild-type mice, protein synthesis levels in the knock-in mice were actually higher, suggesting that RPS6 phosphorylation down-regulates protein synthesis in at least liver cells [12]. Evidence shows that RPS6 phosphorylation plays other key roles, such as regulating translation of 5' terminal oligopyrimidine (TOP) mRNA tracts. Additionally, elevated RPS6 levels were discovered in primary diffuse large B-cell nonHodgkin lymphoma samples with excessive cellular proliferation, whereas genetic treatment with shRPS6 led to a reduction in cell proliferation [14]. Given the potential novel roles that RPS6 may play, further investigation is needed to discern the mechanism by which RPS6 expression may regulate axon growth.

\section{RESESARCH PROBLEM}

Currently unpublished data from Dr. Hetman's lab has shown that shRNA-mediated knockdown of TIF-IA and various ribosomal proteins (RPL4, RPS14, and RPS6) significantly reduced dendritic growth [Slomnicki et al. In preparation]. While working in Dr. Hetman's lab during the previous academic year, we sought to continue this line of research and investigate whether a similar effect is observed in growth of axon. Because axons are a uniquely neuronal appendage, the effects that such disruptive knockdowns have on axons are of particular importance: the unique ability of axons to successfully elongate to great lengths underlies the structure and connectivity of a healthy nervous system. Therefore, this project seeks to investigate the importance of ribosomal biogenesis and nucleolar activity for axon development. In order to do this, ribosomal biogenesis was partially inhibited via shRNA knockdown of RPS6, and nucleolar function was disrupted by strong inhibition of RNA pol I activity via shTIF-IA knockdown.

\section{EXPERIMENTAL PROCEDURES}

Materials: The following antibodies and reagents were obtained from commercial sources: reagents included BDNF (Alomone, Haifa, Israel) and Lipofectamine 2000 (Invitrogen, Carlsbad, CA); primary antibodies included rabbit anti-GFP (MBL; 1/1000) and mouse antiB23/NPM1 (Sigma; 1/750); and secondary antibodies included Alexa Fluor 488 goat anti-rabbit IgG (Invitrogen, 1/300) and Alexa Fluor 555 goat anti-mouse IgG (Invitrogen, 1/300).

Plasmid Preparation: Back transformation allowed for the extraction of the desired pSUPER plasmid vector (OligoEngine) from Escherichia coli bacteria: the cells were subjected to a membrane dissolving chemical and centrifuged, and the resulting supernatant containing the less dense plasmid DNA was collected. A plasmid mini-preparation kit (Qiagen) was then used to chromatographically isolate the DNA, utilizing various buffers to remove contaminants such as RNA, nucleases, and carbohydrates. Following workup, plasmid DNA sample purity was determined using a NanoDrop spectrophotometer.

Neuron Culture \& Transfection: Hippocampal neurons were isolated from newborn SpragueDawley rats (Harlan) at postnatal day zero (P0), as described previously [15]. Briefly, hippocampi were dissected and digested using papain, followed by a trypsin inhibitor; the hippocampi were then triturated in Basal Medium Eagle (BME) supplemented with 10\% heatinactivated bovine calf serum (Hyclone). Finally, the hippocampal neurons were plated at a 
density of $0.2 \times 10^{6}$ per well in a 24 -well plate in Neurobasal medium containing $2 \%$-B27 nutrition supplement (Invitrogen), $1 \mathrm{mM}$ L-glutamine, 100 units $/ \mathrm{mL}$ penicillin, and $0.1 \mathrm{mg} / \mathrm{mL}$ streptomycin. Cells were plated onto poly-D-lysine- and laminin-coated $12 \mathrm{~mm}$ diameter plastic coverslips that were produced in the lab from the electron microscopy-grade myalar masks (Electron Microscopy Sciences). Half of the media was changed every other day. At one day in vitro (DIV 1) neurons were transiently cotransfected with expression plasmids for GFP $(0.1 \mu \mathrm{g}$ of plasmid DNA $/ 2 \times 10^{5}$ neurons) as a transfection marker, and either control Renilla luciferase shRNA (shLuc), shTIF-IA, or shRPS6 $\left(0.4 \mu \mathrm{g}\right.$ of plasmid DNA/2 $\times 10^{5}$ neurons), using Lipofectamine 2000 according to standard manufacturer protocol. On DIV 2 the cells were also treated with either human recombinant BDNF $(20 \mathrm{ng} / \mathrm{mL})$ dissolved in the vehicle solution of $0.1 \% \mathrm{BSA} / \mathrm{PBA}$, or the vehicle alone, for a further 24 hours, followed by fixing cells with $4 \%$ formaldehyde solution after immunostaining on DIV 3-4.

Immunostaining: All cultured neurons were stained after 10-minute fixation in 4\% PFA with 4\% sucrose, followed by washing with PBS. GFP staining was performed using primary rabbit antiGFP antibody (1/1000), followed by secondary Alexa Fluor 488 goat anti-rabbit IgG (1/300). B23 staining was performed using primary mouse anti-B23/NPM1 antibody (1/750), followed by secondary Alexa Fluor 555 goat anti-mouse IgG (1/300). In all cases, standard staining protocols were followed. Following immunostaining, cells were counterstained with $2.5 \mu \mathrm{g} / \mathrm{mL}$ of the DNA dye Hoechst 33258 in order to visualize nuclear morphology. After washing in PBS, coverslips were mounted on coverslips in glycerol gelatin (Sigma) containing p-phenyldiamine glycerol.

shRNA Construct Generation \& Validation: Two different shRPS6 constructs (designated as "shRPS6.1" and "shRPS6.2") and three different shTIF-IA constructs (used together, designated as "shTIF-IA") had been previously generated by analyzing each mRNA sequence using shRNA design software (Ambion.com) and off-target prediction software (sonnhammer.sbc.su.se) as previously described. As previously described, oligonucleotides were designed

(S6\#1: 5'-gatccccgatgatgtccgccagtatgttcaagagacatactggcggacatcatcttttta-3';

S6\#2: 5'-gatccccgattcagcgtcttgttactttcaagagaagtaacaagacgctgaatcttttta-3';

TIF-IA\#1: 5'-gatccccgacttagagttgttgaagattcaagagatcttcaacaactctaagtctttttggaaa-3';

TIF-IA\#2: 5' -gatcccegcacagactgtcttccttattcaagagataaggaagacagtctgtgctttttggaaa-3';

TIF-IA\#3: 5'-gatccccgtgttctgctacaccatcattcaagagatgatggtgtagcagaacactttttggaaa-3') together with their complementary counterparts, annealed and sub-cloned into a pSUPER vector digested with BgIII and HindIII [26; Slomnicki et al. In preparation]. Within the shRPS6 experiment, the first two groups consisted of the separated S6\#1 (shRPS6.1) and S6\#2 (shRPS6.2) sequences, respectively. In order to control for potential off-target effects, the third shRPS6 experimental group consisted of both shRPS6 constructs (S6\#1 and S6\#2) mixed together in halved, proportionate amounts $\left(0.2 \mu \mathrm{g}\right.$ of plasmid DNA $/ 2 \times 10^{5}$ neurons of both constructs together). The control group was transfected with shLuc, which targets the Renilla luciferase mRNA sequence (5'-caaaggaaacggatgataa-3') [26]. Validation of constructs and confirmation of successful RPS6 and TIF-IA knockdown was previously demonstrated [26; Slomnicki et al. In preparation].

Image Acquisition \& Morphometric Analysis: Fluorescence microscopy (Zeiss Observer.Z1 inverted microscope) was used to obtain images from stained and fixed slides. Unfragmented neurons were visualized with GFP immunostaining under a 10x objective lens. AxioVision software was used to obtain black-and-white TIFF image files. Morphometric analysis of TIFF 
images was carried out using the program Neuron Studio to trace axon paths [44]. Tracing files generated by Neuron Studio were then analyzed in the MATLAB program (MathWorks) using custom scripts that calculated the following key axonal parameters; total axon length, longest axon path and number of branches

Quantification of shTIF-IA Inhibition: Nucleolar dispersion was assessed in shTIF-IA-transfected neurons by assessing B23 immunostaining: control shLuc cells and cells with unsuccessful shTIF-IA transfection presented with dense, red, brightly stained B23-positive foci indicative of intact nucleoli; cells with successful shTIF-IA transfection lacked these dense B23-positive foci and instead presented a more diffuse signal, indicating nucleolar dispersion. Cells not indicating this nucleolar dispersion were not considered for imaging, tracing, or statistical analysis.

Statistical Analysis: Statistical analysis of the data involved using a two-way analysis of variance (ANOVA) in conjunction with post hoc Fisher least significant difference tests.

\section{EXPERIMENTAL GROUPS}

\begin{tabular}{|c|c|c|c|}
\hline \multicolumn{4}{|c|}{ shRPS6 Experiments } \\
\hline $\begin{array}{c}\text { Control Group - } \\
\text { shLuc }\end{array}$ & $\begin{array}{c}\text { Experimental Group \#1 - } \\
\text { shRPS6.1 }\end{array}$ & $\begin{array}{c}\text { Experimental Group \#2 - } \\
\text { shRPS6.2 }\end{array}$ & $\begin{array}{c}\text { Experimental Group \#3 - } \\
\text { shRPS6.1 \& shRPS6.2 }\end{array}$ \\
\hline Vehicle & Vehicle & Vehicle & Vehicle \\
\hline Vehicle + BDNF & Vehicle + BDNF & Vehicle + BDNF & Vehicle + BDNF \\
\hline
\end{tabular}

\begin{tabular}{|c|c|}
\hline \multicolumn{2}{|c|}{ shTIF-IA Experiments } \\
\hline Control Group - shLuc & Experimental Group - shTIF-IA \\
\hline Vehicle & Vehicle \\
\hline Vehicle + BDNF & Vehicle + BDNF \\
\hline
\end{tabular}

\section{RESULTS}

First, we set out to determine the effects of transient shRNA knockdown of RPS6 on axon morphology in rat hippocampal neuron cultures. Cultures of transiently transfected and immunostained hippocampal neurons were fixed on slides, after which microscopic images were taken using a GFP filter. Slides were coded to avoid experimenter bias, and axons were consistently identified based on characteristic appearance and length [Figure 2]. Following morphometric analysis with NeuronStudio and MATLAB software, we primarily focused on three axonal parameters: total length, longest path, and number of branches.

For all control and experimental groups involving shRPS6, each group's data represents the mean \pm S.E.M. for a total of at least 39 randomly selected neurons from two independent experiments. Under baseline shLuc control conditions, axons demonstrated the following level of growth and complexity: total axon length of $809 \pm 45 \mu \mathrm{m}$, longest axon path of $442 \pm 17 \mu \mathrm{m}$, and $6.78 \pm 0.67$ axonal branches (all data presented as means \pm S.E.M.) [Figures 3-5]. Upon BDNF stimulation, the shLuc controls did not experience any significant neurotrophic boost of axon 
development compared to the baseline vehicle treatment: total axon length of $820 \pm 40 \mu \mathrm{m}$, longest axon path of $473 \pm 20 \mu \mathrm{m}$, and $6.93 \pm 0.65$ axonal branches (compared to baseline, all three parameters not significant (NS), $p>0.05$ ) [Figures 3-5].

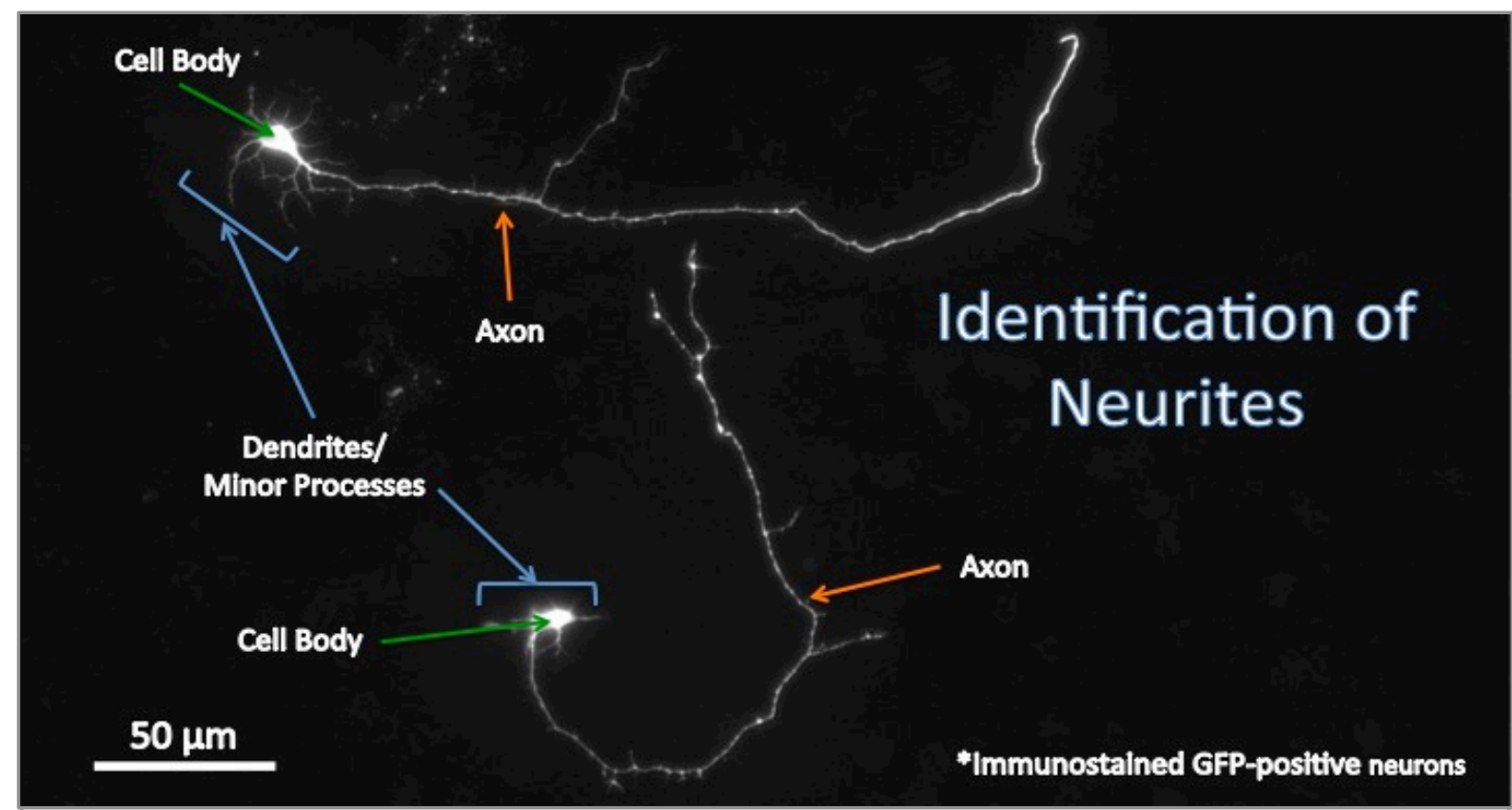

Figure 2: Per the characteristic stages of neurite outgrowth for hippocampal neuron cultures, axons grow much more quickly than dendrites; therefore, by DIV 3-4, their length generally makes axons easy to distinguish from the shorter dendrites [23]. Thus, axons were identifiably distinct due their relatively sizable lengths (as compared to smaller dendrites, which remained clustered closely around the cell body).

Of the three parameters, shRNA treatment only yielded statistically significant differences in total axon length. Total length was reduced (albeit not drastically) in all shRPS6 experimental groups except for the mixed construct group that was exposed to BDNF. Compared to the shLuc control baseline, decreases were seen with mixed shRPS6 constructs ( $809 \pm 45$ vs. $587 \pm 25 \mu \mathrm{m}, p$ $<0.001)$, shRPS6.1 ( $809 \pm 45$ vs. $712 \pm 34 \mu \mathrm{m}, p<0.05)$, and shRPS6.2 (809 \pm 45 vs. $680 \pm 33 \mu \mathrm{m}$, $p<0.01$ ); compared to the shLuc control with BDNF treatment, decreases were only seen with shRPS6. $1(820 \pm 40$ vs. $667 \pm 26 \mu \mathrm{m}, p<0.01)$ and shRPS6.2 $(820 \pm 40$ vs. $699 \pm 30 \mu \mathrm{m}, p<$ $0.05)$, but not with mixed shRPS6 constructs $(820 \pm 40 \mathrm{vs} .773 \pm 33 \mu \mathrm{m}, N S)$ [Figure 3 ]. The results for longest path and number of branches did not indicate a consistent, significant effect on axonal growth. Compared to the shLuc control baseline, the longest axon path was decreased by mixed shRPS6 ( $442 \pm 17$ vs. $370 \pm 15 \mu \mathrm{m}, p<0.01$ ), but was unaffected by shRPS6.1 (442 \pm 17 vs. $445 \pm 20 \mu \mathrm{m}, N S$ ) and shRPS6.2 (442 \pm 17 vs. $401 \pm 17 \mu \mathrm{m}, N S$ ); compared the shLuc control with BDNF, longest axon path saw no effect with mixed shRPS6 (473 \pm 21 vs. $460 \pm 15 \mu \mathrm{m}, N S$ ), a decrease with shRPS6.1 (473 \pm 21 vs. $416 \pm 15 \mu \mathrm{m}, p<0.05$ ), and no effect with shRPS6.2 (473 \pm 21 vs. $443 \pm 18 \mu \mathrm{m}, N S)$ [Figure 4]. Compared to the shLuc control baseline, there was no effect on number of axonal branches with mixed shRPS6 (6.78 \pm 0.67 vs. $5.28 \pm 0.46, N S)$, shRPS6. 1 ( $6.78 \pm 0.67$ vs. $7.30 \pm 0.84, N S)$, or shRPS6. 2 
( $6.78 \pm 0.67$ vs. $5.48 \pm 0.51, N S)$; compared to the shLuc control with BDNF, no effect was seen for mixed shRPS6 ( $6.93 \pm 0.65$ vs. $6.00 \pm 0.55, N S)$ or shRPS6. $(6.93 \pm 0.65$ vs. $6.13 \pm 0.57, N S)$, but there was a decrease with shRPS6.2 (6.93 \pm 0.65 vs. $5.08 \pm 0.58, p<0.05)$ [Figure 5].
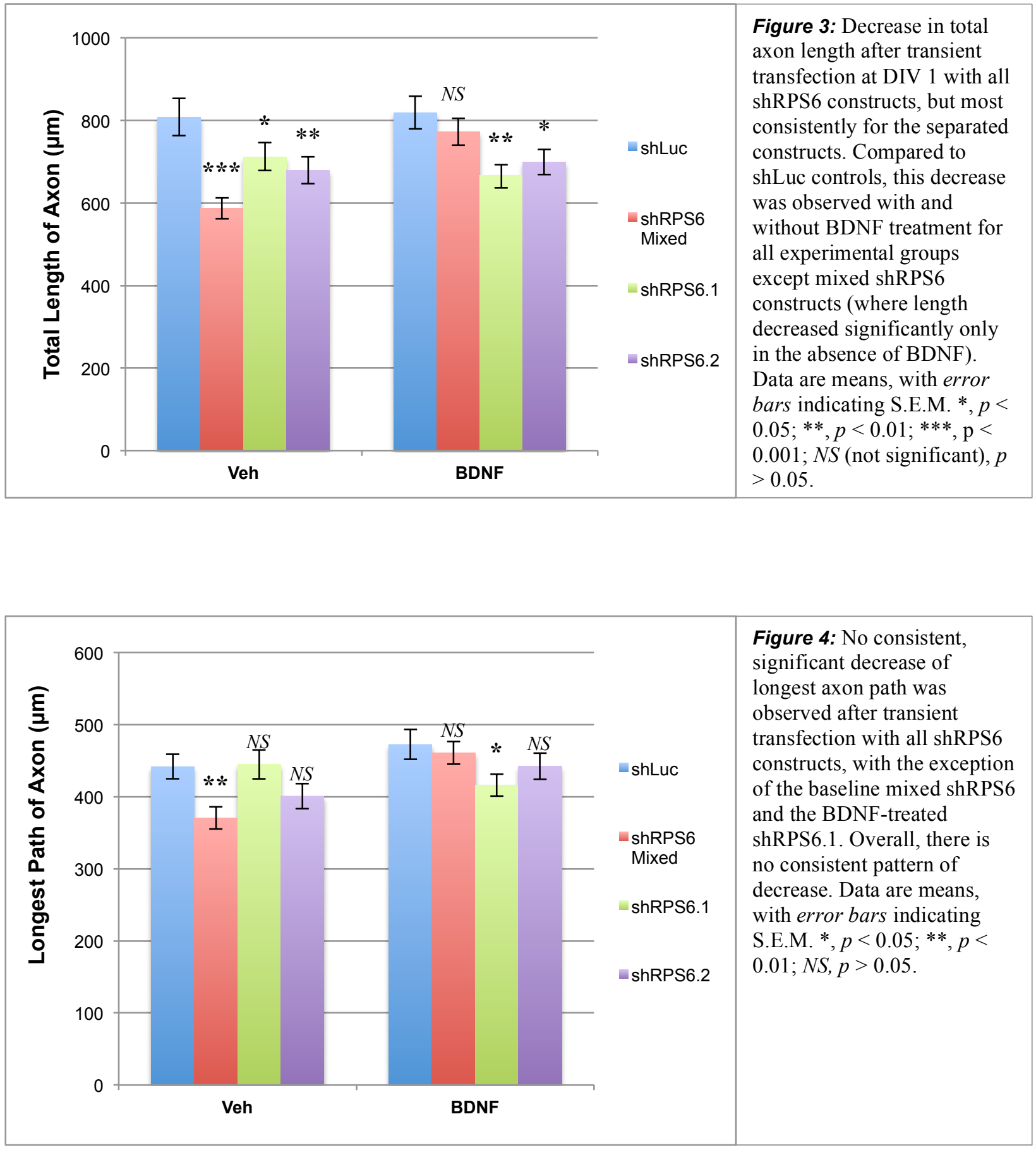


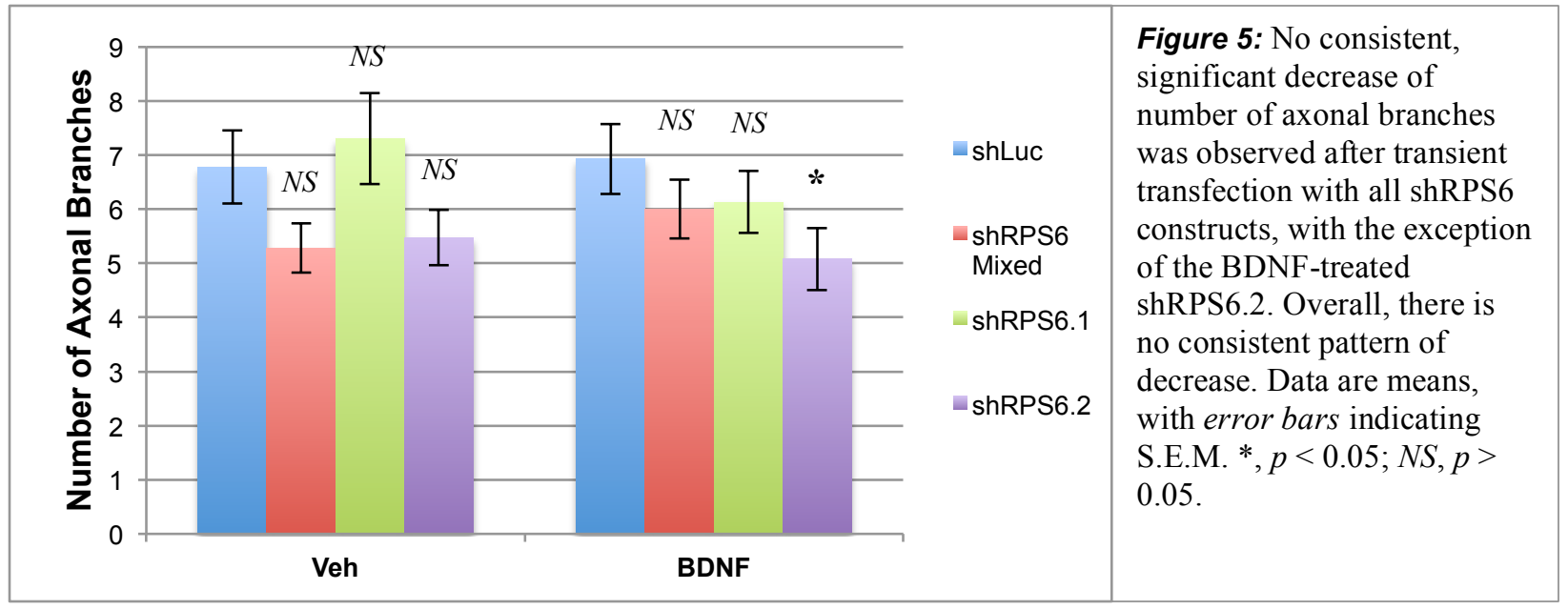

Also, data from axonal experiments with shTIF-IA were examined by selecting specifically for cells that demonstrated complete dispersion of the immuostained nucleolar marker B23, which normally remains bound to nucleolar rRNA [18, 24]. By selecting for cells lacking distinct B23immunostained nuclei, we were able to ascertain exactly which cells had indeed experienced shTIF-IA-mediated inhibition of pol I (and thus inhibition of de novo ribosome synthesis) [Figure 6]. For all shLuc control groups in the shTIF-IA experiment, the data represents the mean \pm S.E.M. for a total of at least 39 randomly selected neurons from two independent experiments. For all shTIF-IA experimental groups, the data represents the mean \pm S.E.M. for a total of at least 19 randomly selected neurons (among those that demonstrated B23 dispersion) from two independent experiments.

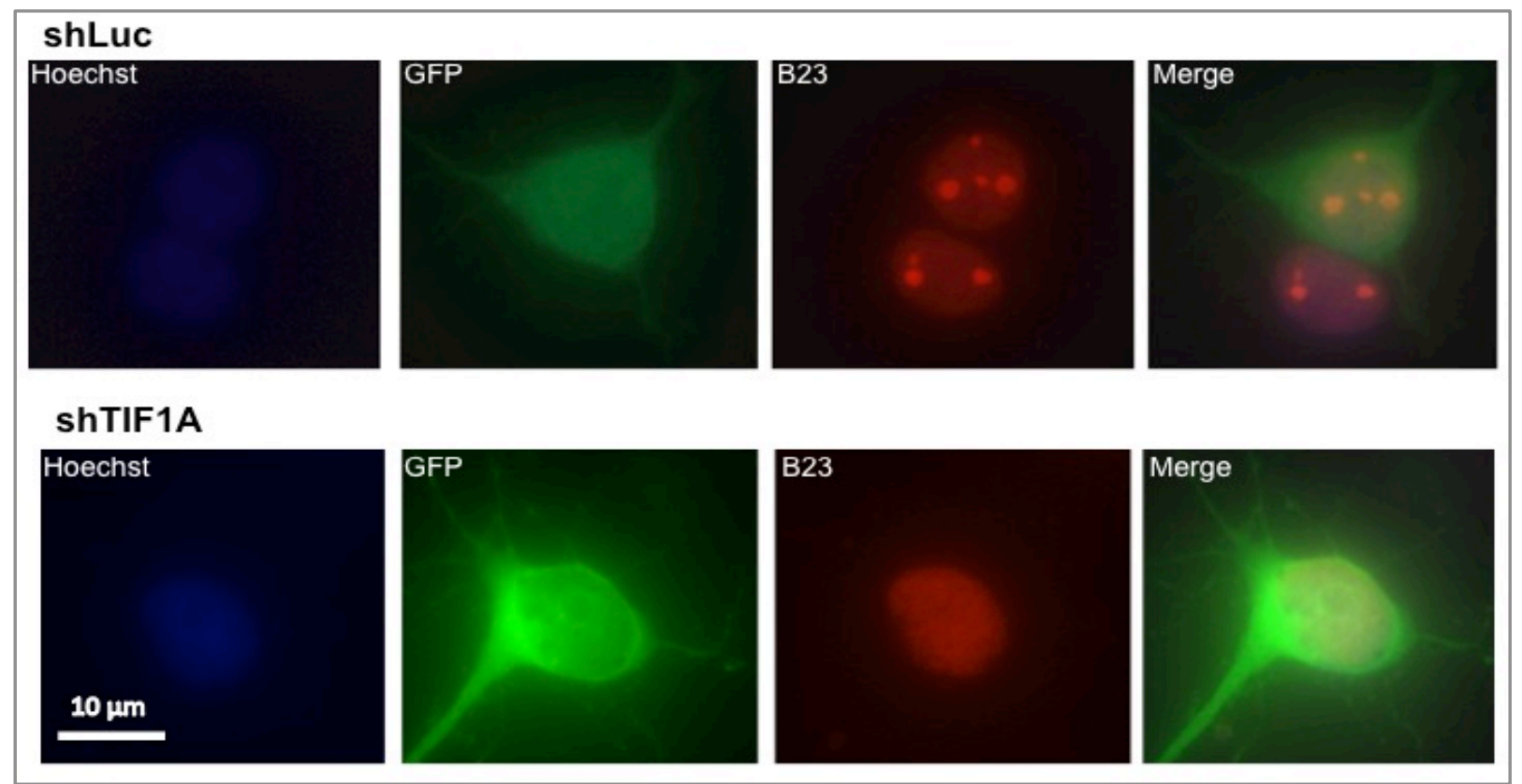

Figure 6: Immunostaining with fluorescence microscopy allowed for selection of only those neurons that demonstrated dispersion of the nucleolar phosphoprotein marker B23 and, thus, successful transfection by shTIF-IA. In shLuc control cells, B23 staining resulted in small, dense, bright red granules (nucleoli) within a larger, less dense, dull red region (nucleus). In shTIF-IA cells, B23 staining resulted in the absence of any of the smaller, dense, bright red granules, thereby indicating nucleolar dispersion. 
Under baseline shLuc control conditions, axons demonstrated the following level of growth and complexity: total axon length of $558 \pm 28 \mu \mathrm{m}$, longest axon path of $382 \pm 13 \mu \mathrm{m}$, and $4.18 \pm 0.45$ axonal branches. Once again, BDNF treatment of shLuc controls failed to stimulate increased growth compared to the baseline treatment: total axon length of $617 \pm 32 \mu \mathrm{m}$, longest axon path of $408 \pm 25 \mu \mathrm{m}$, and $5.03 \pm 0.70$ axonal branches (compared to baseline, all three parameters $N S$, $p>0.05)$. Within baseline and BDNF treatment groups for each parameter, shTIF-IA did not result in a statistically significant decrease for any parameters, with the only exception being a decrease in branch ratio under BDNF treatment. Compared to the baseline shLuc control, shTIFIA treatment produced no change in total axon length under baseline conditions (558 \pm 28 vs. $589 \pm 29 \mu \mathrm{m}, N S)$ or BDNF treatment $(617 \pm 32$ vs. $512 \pm 33 \mu \mathrm{m}, N S)$ [Figure 7]; similarly, longest axon path saw no change under baseline conditions ( $382 \pm 13$ vs. $341 \pm 16 \mu \mathrm{m}, N S)$ or BDNF treatment ( $408 \pm 25$ vs. $334 \pm 19 \mu \mathrm{m}, N S$ ) [Figure 8]; and the number of axonal branches saw no change under baseline conditions $(4.18 \pm 0.45$ vs. $5.39 \pm 0.67 \mu \mathrm{m}, N S)$ but decreased under BDNF treatment $(5.03 \pm 0.70$ vs. $3.53 \pm 0.48 \mu \mathrm{m}, p<0.05)$ [Figure 9].

All in all, these data indicate that shRPS6 did result in some significant decreases across all parameters of axon growth, though overall the effects were relatively small and somewhat inconsistent. The shTIF-IA produced essentially no significant decrease of axon growth, with the only exception being the decrease in number of axonal branches under BDNF treatment. Therefore, ribosomal biogenesis does not appear to be of major importance for axon growth at least during early phases of this process.
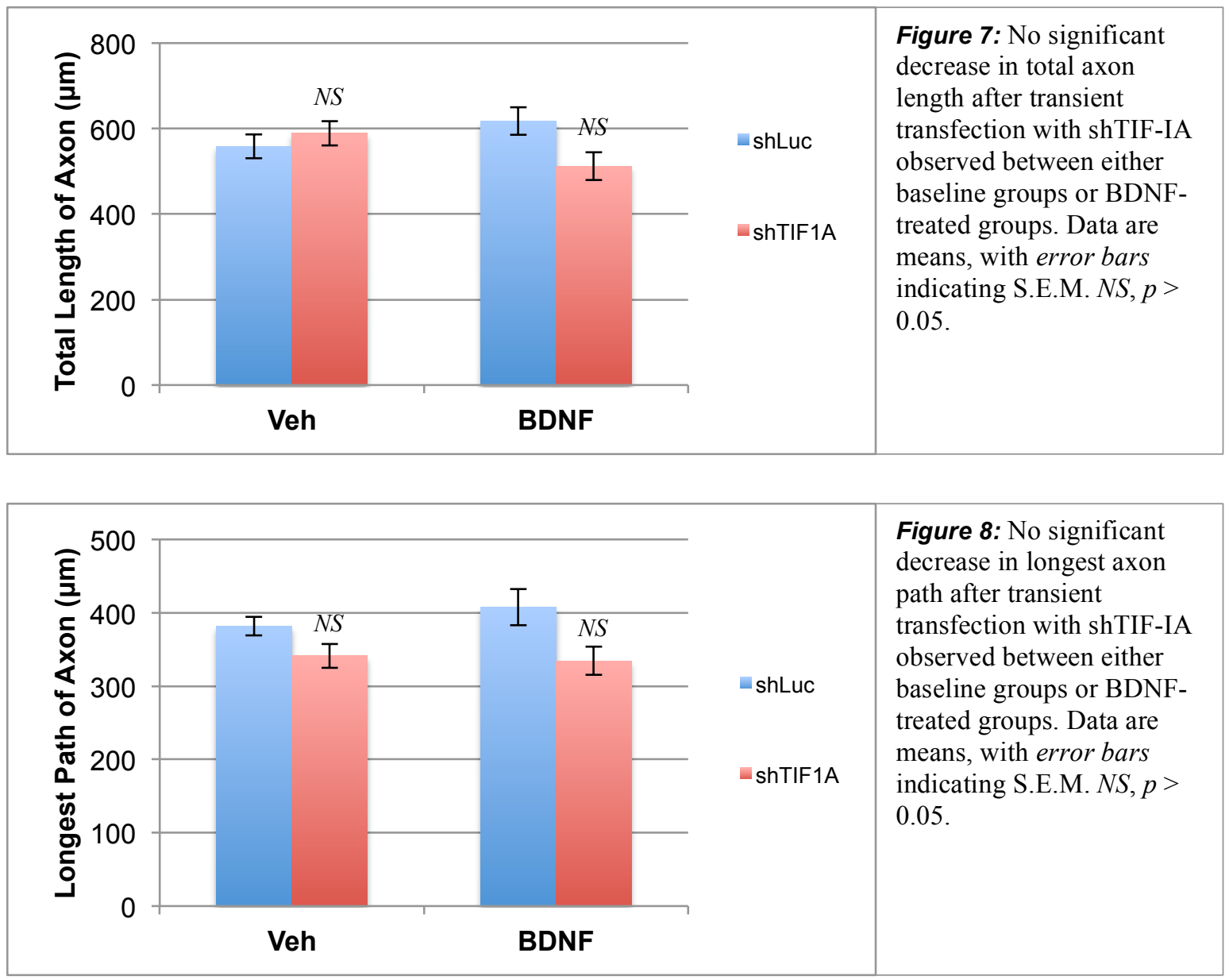


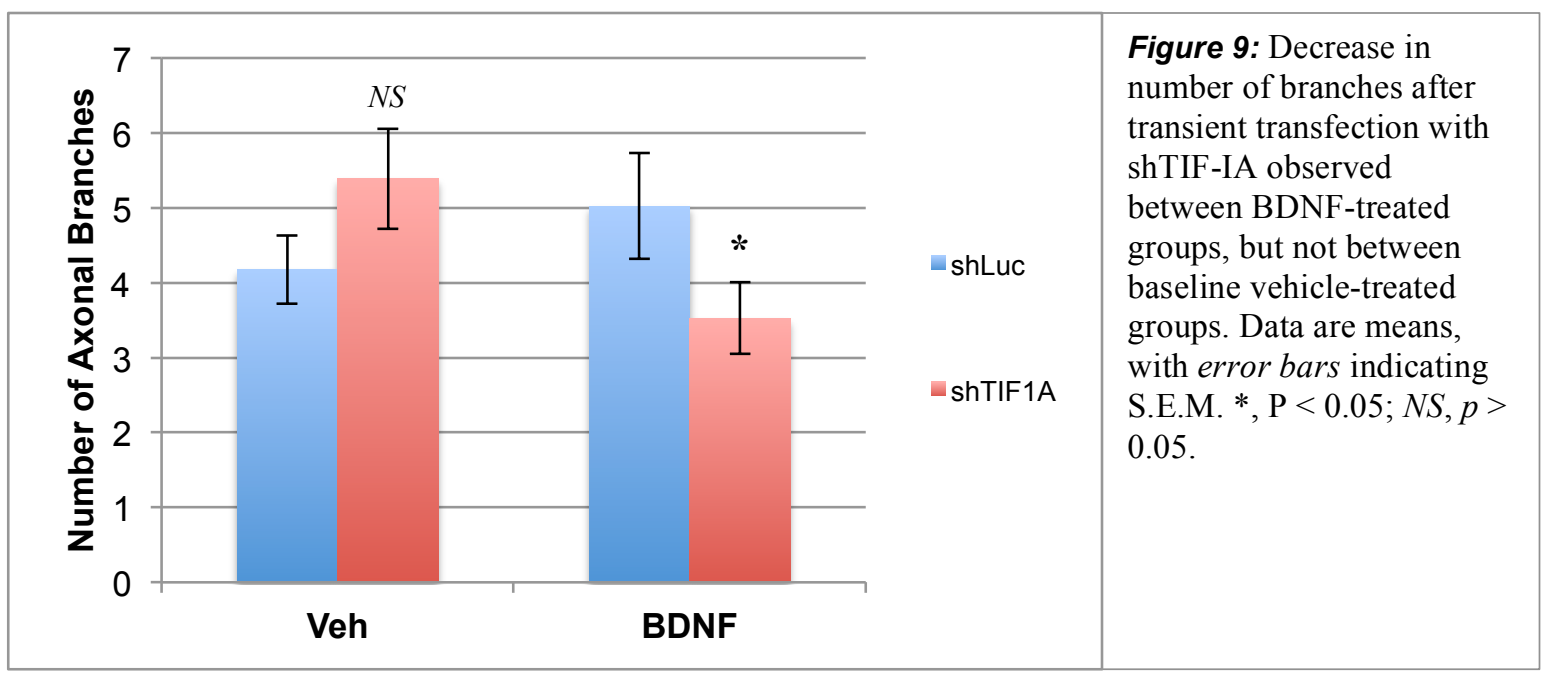

\section{DISCUSSION}

Initially, our hypothesis held that shRNA-mediated knockdown of TIF-IA and RPS6 would result in a decrease in axonal growth due to direct inhibitory effects on RNA pol I activity and ribosomal biogenesis. Ultimately, however, our data indicate that this hypothesis was incorrect. Despite antagonizing TIF-IA and RPS6 - both of which play important roles in ribosome production - it appears that pre-existing ribosomes are sufficient for initial axonal elongation [Figure 10].

However, the duration of this pre-existing ribosome supply would presumably be temporally limited by the 6-day half-life of cytoplasmic ribosomes in the rat brain [9]. Thus, given more time, ribosomal biogenesis inhibition would be expected to affect translation and axonal growth. Aside from the hypothetical aspect regarding pre-existing ribosome supply, this schematic also assumes the implicit necessity of protein synthesis for axonal growth during this stage; this assumption is supported, however, by previously mentioned research showing that CHXmediated disruption of protein synthesis did indeed inhibit axonal growth during DIV 1-2 [42].

To be sure, shRNA knockdown of RPS6 did have a consistent, slight inhibitory effect on total axonal length (but not on axon path or number of axonal branches) during the initiation phase of 
axonal development. Yet, while the decreases in total axon length for the shRPS6 constructs were statistically significant, the effect was relatively small and not entirely consistent. These results were corroborated by our experiments with shTIF-IA when B23 dispersion was taken into consideration to assure shTIF-IA transfection and inhibition of rRNA transcription: the data did not show a consistent, significant decrease in any of the three morphogenetic parameters. Taken together, these complementary findings indicate that ribosome biogenesis and RNA pol I activity are not important for axonal growth during the initiation phase of axonal development. When interpreting these results, the slight inhibitory effect of shRPS6 could be attributed to the role of RPS6 in ribosomal biogenesis, but could also potentially indicate that RPS6 has a noncanonical role other than being a structural and functional ribosome component. Once again, future experiments would be necessary to obtain more evidence. The relatively modest magnitude of the observed inhibitory effects, however, call into question the utility and effectiveness of further experiments, such as with phosphomutants to block RPS6 phosphorylation.

Interestingly, there is no significant difference in the results for axon morphological parameters between the baseline vehicle groups and the BDNF treatment groups. Despite the fact that the vehicle treatment ideally represents baseline growth while BDNF treatment represents neurotrophically-enhanced growth, it appears that BDNF is not significantly increasing axon elongation during this stage. Given that the cells were fixed by DIV 3-4, this lack of BDNF stimulation can likely be explained by the considerably robust level of axon growth around DIV 2 [23]; treatment with BDNF does little to increase this already robust, early growth.

Future research would include an investigation of the importance of ribosomal biogenesis and pol I activity during later phases of axonal development. Presumably, both prolonged depletion of ribosomes and nucleolar disruption would result in a more extreme deficit during later phases. As such, it is probable that inhibition of ribosomal biogenesis (via ribosomal protein knockdown) and inhibition of pol I activity (via TIF-IA knockdown) during later phases would result in even more pronounced inhibition of axonal growth. Conducting experiments of this nature, however, is difficult. Such experiments would require waiting until at least DIV 6-9, at which point the axons grow to unwieldy lengths and present problems for tracing and morphometric analysis.

Within a broader context, this line of research aims to elucidate the role of ribosomal biogenesis in healthy nervous system development at the cellular level. A relatively neglected field of study has been the intrinsic mechanisms underlying neuronal development, with much emphasis being placed instead on stimulated or altered growth and pathological growth problems. As such, this research on rRNA and ribosomal production complements prior research on the importance of biosynthesis of other macromolecules for normal neuron development during this initial growth phase, including the processes of protein synthesis, lipid synthesis, and general transcription. Additionally, it explores how dysfunctional ribosomal biogenesis or irregular neurite morphogenesis may contribute to the pathogenesis of nervous system disorders. Generally, it is expected that a lack of mature, functional ribosomes - which are responsible for protein translation and, in turn, cell growth - would be lethal during embryonic development. However, a recently investigated class of rare, genetic diseases of ribosome function and biogenesis referred to as "ribosomopathies" - has shown this expectation is not always true $[19,20]$. Among the causes for these ribosomopathies is a deficiency of ribosomal proteins: Diamond-Blackfan 
anemia is caused by congenital mutations in the genes for RPS19 and other ribosomal proteins, and 5q- syndrome is brought on in part by a haploinsufficiency of RPS14 [20]. As a more neurologically relevant example, one study has provided evidence that links a decline in protein synthesis both to a decline in ribosome precursors and to high levels of oxidative damage to ribosomal-associated RNA in the brains of patients with Alzheimer's disease [21]. In addition, mutations in the gene for RPL10 have been identified as the potential causal agents of the cognitive malfunction seen in various cases of autism [22]. Finally, another recent study has identified RPS15 as a mediator of the LRRK2-induced neurodegeneration seen in Parkinson's disease [25]. As the involvement of dysfunctional ribosomal biogenesis and neurite morphogenesis in various diseases becomes increasingly apparent, our knowledge of these cellular processes will continue to grow in importance.

In conclusion, neither shRPS6 nor shTIF-IA knockdown produced a consistent, meaningful inhibitory effect on axon growth. It can be argued that the lack of a large inhibitory effect following shRPS6 treatment is attributable to the fact that such shRNA knockdown is only able to bring about an incomplete knockdown of RPS6, which is merely a single ribosomal component. Consequently, the shTIF-IA experiment becomes increasingly important because it avoids this issue: selecting for cells that only demonstrated complete B23 dispersion allowed for the incorporation of only those cells whose nucleoli had been disrupted. Because nucleoli are essentially brought into existence by their very "act of building a ribosome" [27], this lack of visible nucleoli indicates that the entire process of ribosomal biogenesis has been disrupted. Therefore, combining the results from these two experiments provides convincing evidence that nucleolar RNA pol I activity (and the nucleolus-based process of ribosomal biogenesis) are of minor importance to axon growth during this early phase of development. As an explanation for this ability of axons to continue growing in the face of such inhibition, it is important to consider the fact that shTIF-IA was shown to not disrupt translation specifically during the timeframe of DIV 1-3 in which these experiments were conducted; previous experiments in which both dendrite growth and translational capacity were diminished by shTIF-IA were conducted later during DIV 6-9 [Slomnicki and Hetman, unpublished observations]. With this consideration in mind, it appears that the various effects of ribosomal biogenesis inhibition on neurites can be explained by differential modulation of protein synthesis, which appears to be essential for axon growth [42]. Furthermore, considering the 6-day half-life of cytoplasmic ribosomes in the rat brain and the robustness of cellular growth in this early stage $[9,23]$, the cell is able to maintain protein synthesis and axon development despite experimental disruption of de novo ribosomal biogenesis.

\section{REFERENCES}

1. Schwarzacher HG, Wachtler F. The nucleolus. Anat Embryol (Berl). 1993 Dec;188(6):515-36.

2. Sirri V, Urcuqui-Inchima S, Roussel P, Hernandez-Verdun D. Nucleolus: the fascinating nuclear body. Histochem Cell Biol. 2008 Jan;129(1):13-31.

3. Wilson DN, Doudna Cate JH. The structure and function of the eukaryotic ribosome. Cold Spring Harb Perspect Biol. 2012;14(5).

4. Green R, Noller H.F. Ribosomes and translation. Annu Rev Biochem. 1997;66:679-716. 
5. Hernandez-Verdun D, Roussel P, Thiry M, Sirri V, Lafontaine DL. The nucleolus: structure/function relationship in RNA metabolism. Wiley Interdiscip Rev RNA. 2010 Nov-Dec;1(3):415-31.

6. Lee MG, Van der Ploeg LH. Transcription of protein-coding genes in trypanosomes by RNA polymerase I. Annu Rev Microbiol. 1997;51:463-89.

7. Gomes C, Smith SC, Youssef MN, Zheng JJ, Hagg T, Hetman M. RNA polymerase 1driven transcription as a mediator of BDNF-induced neurite outgrowth. J Biol Chem. 2011 Feb 11;286(6):4357-63.

8. Hetman M, Pietrzak M. Emerging roles of the neuronal nucleolus. Trends Neurosci. 2012 May;35(5):305-14.

9. Stoykova AS, Dudov KP, Dabeva MD, Hadjiolov AA. Different rates of synthesis and turnover of ribosomal RNA in rat brain and liver. J Neurochem. 1983 Oct;41(4):942-9.

10. Gressner AM, Wool IG. The phosphorylation of liver ribosomal proteins in vivo: Evidence that only a single small subunit protein (S6) is phosphorylated. J Biol Chem. 1974;249:6917-6925.

11. Krieg, J. et al. (1988) Identification of the 40 S ribosomal protein S6 phosphorylation sites induced by cycloheximide. J. Biol. Chem. 263, 11473-11477.

12. Ruvinsky I, Meyuhas O. Ribosomal protein S6 phosphorylation: from protein synthesis to cell size. Trends Biochem Sci. 2006 Jun;31(6):342-8.

13. Nygård $\mathrm{O}$, Nilsson L. Translational dynamics. Interactions between the translational factors, tRNA and ribosomes during eukaryotic protein synthesis. Eur J Biochem. 1990 Jul 20;191(1):1-17.

14. Hagner PR, Mazan-Mamczarz K, Dai B, Balzer EM, Corl S, Martin SS, Zhao XF, Gartenhaus RB. Ribosomal protein S6 is highly expressed in non-Hodgkin lymphoma and associates with mRNA containing a 5 ' terminal oligopyrimidine tract. Oncogene. 2011 Mar 31;30(13):1531-41.

15. Habas A, Kharebava G, Szatmari E, Hetman M. NMDA neuroprotection against a phosphatidylinositol-3 kinase inhibitor, LY294002 by NR2B-mediated suppression of glycogen synthase kinase-3beta-induced apoptosis. J Neurochem. 2006 Jan;96(2):335-48.

16. Jaworski J, Biedermann IW, Lapinska J, Szklarczyk A, et al. Neuronal excitation-driven and AP-1-dependent activation of tissue inhibitor of metalloproteinases-1 gene expression in rodent hippocampus. J Biol Chem. 1999 Oct 1;274(40):28106-12.

17. Schneider-Poetsch, T. et al. Inhibition of eukaryotic translation elongation by cycloheximide and lactimidomycin. Nature Chem Biol. 2010 Jan;6:209-217.

18. Okuwaki M. The structure and functions of NPM1/nucleophosmin/B23, a multifunctional nucleolar acidic protein. J Biochem. 2008 Apr;143(4):441-8.

19. Freed EF, Bleichert F, Dutca LM, Baserga SJ. When ribosomes go bad: diseases of ribosome biogenesis. Mol Biosyst. 2010 Mar;6(3):481-493.

20. Narla A, Ebert BL. Ribosomopathies: human disorders of ribosome dysfunction. Blood. 2010 Apr;115(16):3196-3205.

21. Ding Q, Markesbery WR, Cecarini V, Keller JN. Decreased RNA, and increased RNA oxidation, in ribosomes from early Alzheimer's disease. Neurochem Res. 2006 May;31(5):705-10.

22. Klauck SM, Felder B, Kolb-Kokocinski A, et al. Mutations in the ribosomal protein gene RPL10 suggest a novel modulating disease mechanism for autism. Mol Psychiatry. 2006;11(12):1073-1084. 
23. Dotti CG, Sullivan CA, Banker GA. The establishment of polarity by hippocampal neurons in culture. J Neurosci. 1988 Apr;8(4):1454-68.

24. Spector DL, Ochs RL, Busch H. Silver staining, immunofluorescence, and immunoelectron microscopic localization of nucleolar phosphoproteins B23 and C23. Chromosoma. 1984;90(2):139-48.

25. Martin I, Kim JW, Lee BD, Kang H, Xu J-C, Jia H, Stankowski J, Kim MS, et al. Ribosomal protein s15 phosphorylation mediates LRRK2 neurodegeneration in Parkinson's disease. Cell. 2014 Apr 10;157(2):472-85.

26. Kalita K, Makonchuk D, Gomes C, Zheng J, Hetman M. Inhibition of nucleolar transcription as a trigger for neuronal apoptosis. J Neurochem. 2008 Jun 1;105(6):22862299.

27. Mélèse T, Xue Z. The nucleolus: an organelle formed by the act of building a ribosome. Curr Opin Cell Biol. 1995 Jun 7;3(3):319-24.

28. Bartlett WP, Banker GA. An electron microscopic study of the development of axons and dendrites by hippocampal neurons in culture. I. Cells which develop without intercellular contacts. J Neurosci. 1984 Aug;4(8):1944-53.

29. Hay N, Sonenberg N. Upstream and downstream of mTOR. Genes Dev. 2004 Aug $15 ; 18(16): 1926-45$.

30. Grummt I. Life on a planet of its own: regulation of RNA polymerase I transcription in the nucleolus. Genes Dev. 2003 July 15;17(14):1691-702.

31. Zhao J, Yuan X, Frödin M, Grummt I. ERK-dependent phosphorylation of the transcription initiation factor TIF-1A is required for RNA polymerase I transcription and cell growth. Mol Cell. 2003 Feb;11(2):405-13.

32. Mayer C, Zhao J, Yuan X, Grummt I. mTOR-dependent activation of the transcription initiation factor TIF-1A links rRNA synthesis to nutrient availability. Genes Dev. 2-4 Feb 15;18(4):423-34.

33. Alvarez J, Giuditta A, Koenig E. Protein synthesis in axons and terminals: significance for maintenance, plasticity, and regulation of phenotype. With a critique of slow transport theory. Prog Neurobiol. 2000 Sept;62(1):1-62.

34. Obara I, Hunt SP. Axonal protein synthesis and the regulation of primary afferent function. Dev Neurobiol. 2104 Mar;74(3):269-78.

35. Lin AC, Holt CE. Function and regulation of local axonal translation. Curr Opin Neurobiol. 2008 Feb;18(1):60-68.

36. Kirkland RA, Franklin JL. Rate of neurite outgrowth in sympathetic neurons is highly resistant to suppression of protein synthesis: role protein degradation/synthesis coupling. Neurosci Lett. 2007 Jan 3;411(1):52-55.

37. Viktorovskaya OV, Schneider DA. Functional divergence of eukaryotic RNA polymerases: unique properties of RNA polymerase I suit its cellular role. Gene. 2015 Feb 1;556(1):19-26.

38. Schnapp A, Pfleiderer C, Rosenbauer H, Grummt I. A growth dependent transcription initiation factor (TIF-IA) interacting with RNA polymerase I regulates mouse ribosomal RNA synthesis. EMBO J. 1990 Sept;9(9):2857-63

39. Yamamoto RT, Nogi Y, Dodd JA, Nomura M. RRN3 gene of Saccharomyces cerevisiae encodes an essential RNA polymerase I transcription factor which interacts with the polymerase independently of DNA template. EMBO J. 1996 August 1;15(1):3964-73. 
40. Bodem J, Dobreva G, Hoffmann-Rohrer U, Iben S, Zentgraf H, Delius H, Vingron M, Grummt I. TIF-IA, the factor mediating growth-dependent control of ribosomal RNA synthesis, is the mammalian homolog of yeast Rrn3p. EMBO Rep. 2000 Aug;1(2):171-5.

41. Nguyen LT, Mitchell BS. Akt activation enhances ribosomal RNA synthesis through casein kinase II and TIF-IA. Proc Natl Acad Sci USA. 2013 Dec 17;110(51):20681-6.

42. Jareb M, Banker G. Inhibition of axonal growth by brefeldin A in hippocampal neurons in culture. J Neurosci. 1997 Dec 1;17(23):8955-63.

43. Sotelo-Silveira JR, Calliaria A, Cárdenas M, Koenig E, Sotelo JR. Myosin Va and kinesin II motor proteins are concentrated in ribosomal domains (periaxoplasmic ribosomal plaques) of myelinated axons. J Neurobiol. 2004 Aug;60(2):187-96.

44. Wearne SL, Rodriguez A, Ehlenberger DB, Rocher AB, Henderson SC, Hof PR. New techniques for imaging, digitization and analysis of three-dimensional neural morphology on multiple scales. Neuroscience. 2005;136(3):661-80. 